\title{
VIEWPOINT
}

\section{PTCA versus CABG: a different interpretation of the results of randomised trials comparing both treatments}

\author{
N Danchin, P Urban
}

Service de

Cardiologie, $\mathrm{CHU}$

Nancy-Brabois, 54500

Vandoeuvre-lès-Nancy,

France

N Danchin

La Tour Hospital, 1 Avenue JD Maillard, 1217 Meyrin-Genève, Switzerland

P Urban

Correspondence to: Service de Cardiologie, $\mathrm{CHU}$ Nancy, Hôpitaux de Brabois, Rue du Morvan, F- 54511

Vandoeuvre-lès-Nancy email:n.danchin@ chu-nancy.fr

Accepted for publication Professor N Danchin, CEDEX, France. 17 June 1999

The choice of the most appropriate mode of myocardial revascularisation remains open in many patients. All randomised trials comparing surgery (CABG) and angioplasty (PTCA) have shown that both modalities are equivalent in terms of survival or infarct free survival; but all showed that patients treated with PTCA required many more admissions for additional revascularisation procedures during follow up. It was suggested that patients be informed at the time of their initial angiography that the PTCA option would mean more subsequent hospitalisations. The need for reintervention can rightly be seen as one of the major limitations of any revascularisation procedure. It is a significant inconvenience for the patient, increases the time away from a normal active life, and is associated with increased costs. In the BARI ((bypass angioplasty revascularization investigation) trial, ${ }^{1} 54 \%$ of patients randomised to the PTCA arm had hospital admissions for repeat revascularisation procedures, compared with only $8 \%$ in the CABG group. The respective figures in a meta-analysis of the first randomised trials of PTCA $v$ CABG, were $33.7 \%$ and $3.3 \%$. $^{2}$ These results, however, were derived from prospective trials during which angioplasty and coronary bypass procedures were performed after initial hospitalisation for

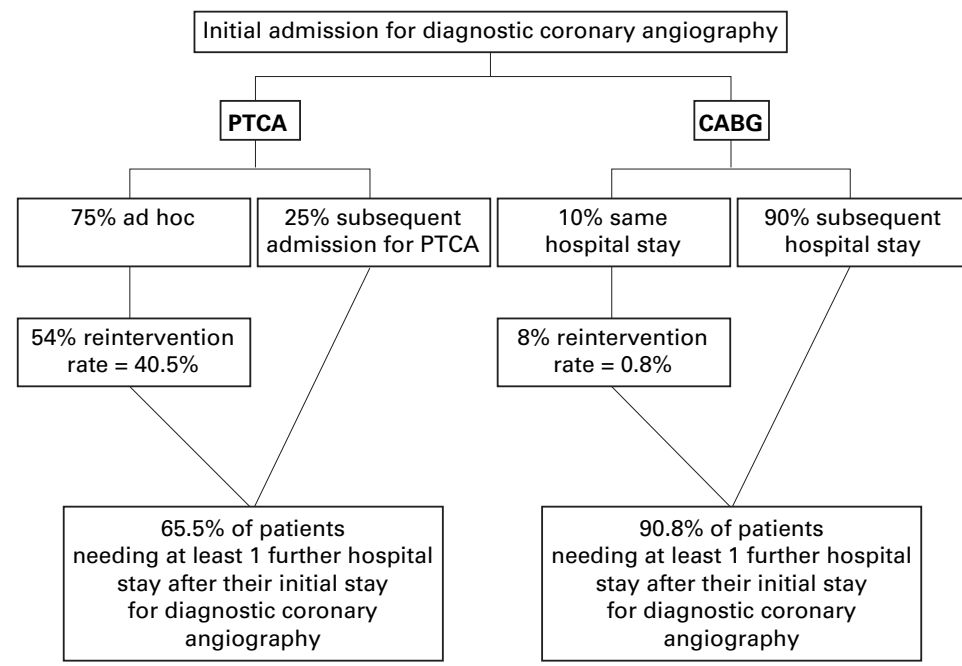

Figure 1 Percentages of new hospital admissions after the initial admission for diagnostic coronary angiography in patients treated with either PTCA or CABG, based on an initial rate of $75 \%$ for ad hoc PTCA (figures derived from the BARI trial). ${ }^{1}$ diagnostic coronary angiography; such an approach does not reflect current practice in many institutions.

Indeed, in recent years, more and more catheterisation laboratories have been performing PTCA in the same session as the diagnostic angiography, the so called ad hoc procedures. ${ }^{3}$ Typically, the patient undergoes coronary angiography, the films are reviewed by an interventional cardiologist and, whenever necessary, discussed with a cardiac surgeon; the decision reached is explained and discussed with the patient, and angioplasty is performed on the same day, usually during the same session. The whole hospital stay is therefore usually no longer than that required for the diagnostic procedure alone, the patient only undergoes a single arterial puncture, and he or she does not have to return to the hospital for the revascularisation procedure. For the patient, a single intervention has taken place, and the cost is obviously much less than would have been engendered by a subsequent hospital admission. In contrast, coronary surgery is seldom performed during the same admission as diagnostic coronary angiography, and therefore usually requires an additional hospital stay compared with ad hoc angioplasty. For the year 1995 , over $75 \%$ of the angioplasty procedures performed at both our hospitals were ad hoc, while fewer than $10 \%$ of coronary bypass operations were performed during the same hospital stay as that of diagnostic angiography.

Therefore, if we compare CABG and PTCA from the time of the initial diagnostic coronary angiography, the need for at least one subsequent hospital admission over five years can be calculated as shown in fig 1 .

In the AQUA (audit and quality control in angioplasty) registry, which tracks PTCA procedures performed in 25 centres randomly selected in five European countries, the rate of ad hoc procedures was $47 \% .{ }^{4}$ Using the BARI estimates of repeat interventions, the expected rate of one or more hospital admissions after the initial admission for coronary angiography would therefore be $78.3 \%$, compared with $90.8 \%$ in patients treated surgically. These figures, which reflect common practice for PTCA in many European countries, should be kept in mind when extrapolating the results of the 
randomised trials of PTCA $v$ CABG, both from the patient's and from the health economist's points of view. Furthermore, the wider usage of intracoronary stents will most likely result in a decrease of the figures of new hospital admissions after the initial revascularisation procedure reported in the BARI trial.

From the physician's point of view, it is entirely appropriate to differentiate a planned staged approach (that is first admission for coronary angiography and second admission for percutaneous revascularisation) from treatment failure requiring a repeat intervention. However, it should be appreciated that the subtleties of such nuances may be lost on the patient, for whom one more hospital admission is often just that: one more hospital admission.

1 The Bypass Angioplasty Revascularization Investigation (BARI) Investigators. Comparison of coronary bypass surgery with angioplasty in patients with multivessel disease. $N$ gery with angioplasty in patien

2 Pocock SJ, Henderson RA, Rickards AF, et al. Meta-analysis of randomised trials comparing coronary angioplasty with bypass surgery. Lancet 1995;346:1184-9.

3 Röthlisberger C, Meier B, on behalf of the Working Group on Coronary Circulation of the European Society of Cardiology. Coronary interventions in Europe in 1992. Eur Heart f1 1995;16: 922-9.

4 Maier W, Endezlin M, Bonzel T, et al. Audit and quality control in angioplasty in Europe: procedural results of the AQUA study 1997. Assessment of 250 randomly selected coronary interventions performed in 25 centers of 5 European countries. Eur Heart F. [In press.] 